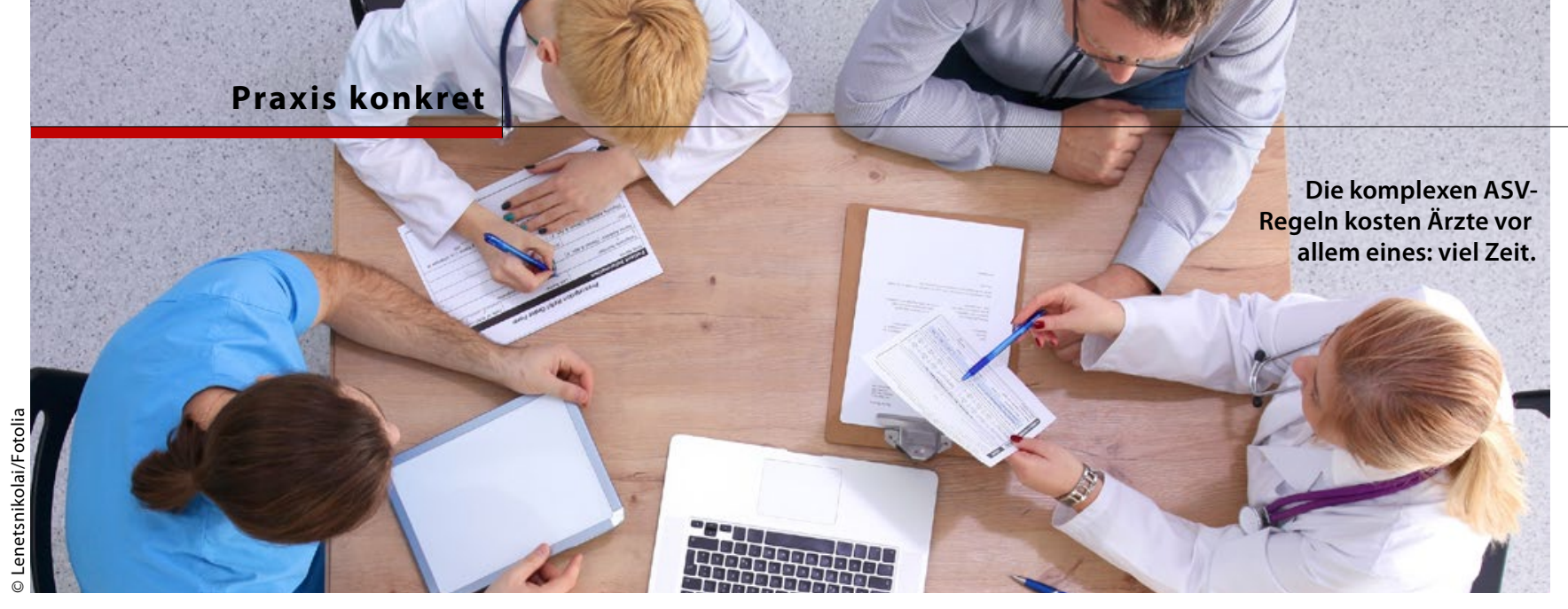

Ambulante spezialfachärztliche Versorgung

\title{
Viel Bürokratie, wenig Ertrag - Ärzte hadern mit der ASV
}

\author{
Vor fünf Jahren wurde die ambulante spezialfachärztliche Versorgung \\ eingeführt. Ärzte, die sich daran beteiligen, benötigen offenbar vor \\ allem eins: viel Enthusiasmus.
}

ünf Jahre nach der Einführung stehen Vertreter von Kassen, Kassenärztlicher Bundesvereinigung (KBV) und Krankenhäusern der ambulanten spezialfachärztlichen Versorgung (ASV; $§ 116$ b neu) überwiegend skeptisch gegenüber. „Die ASV-Richtlinien sind zu lang, komplex und ihre Umsetzung zu zeitaufwendig“, kritisierte beispielsweise Dr. Wulf-Dietrich Leber vom GKV-Spitzenverband bei einem Symposium in Berlin.

Ähnlich bewertete der Hauptgeschäftsführer der Deutschen Krankenhausgesellschaft (DKG) Georg Baum das ASV-Regelwerk. Die Überführung der alten ASV-Verträge in das neue Konstrukt werde gut und gerne zehn Jahre in Anspruch nehmen. Baum: „Alle alten Verträge sollten ohne Nachweis in die neue ASV aufgenommen werden.“

\section{Umsetzung schleppend}

Auch seitens der Patienten war wenig Gutes zu hören. Renate Pfeiffer von der Bundesarbeitsgemeinschaft (BAG) Selbsthilfe kritisierte, dass das ASV-Regelwerk auf wenige Indikationen beschränkt und nicht mit der Aufnahme neuer Erkrankungen zu rechnen sei. Durch die schleppende Umsetzung sei nicht abzuschätzen, ob in absehbarer Zeit ein Nutzen erkennbar ist.

62 Spezialistenteams sind beim ASVBundesverband derzeit bekannt. 41 setzen die ASV für Patienten mit gastrointestinalen Tumoren, 21 jene für Patienten mit Tuberkulose (Tbc) um. Teams für die Versorgung gynäkologischer Tumoren würden derzeit zusammengestellt, teilte der Bundesverband mit.

In den ASV-Teams sind mehrheitlich sowohl Vertrags- als auch Klinikärzte vertreten. Die Teamleiter sehen in der interdisziplinären Versorgung, der intensivierten Betreuung, der sektorenübergreifenden Zusammenarbeit und der kurzfristigen Wahrnehmung von Facharztterminen überwiegend einen Zusatznutzen für die Patienten im Vergleich zu den bisherigen Standards. Das geht aus einer ersten Auswertung der Begleitstudie zur Einführung der ASV hervor.

Die Studie wird von der Berliner Hochschule BBW, dem ASV-Bundesverband und dem Bundesverband Managed Care betreut. Verwertbare Daten lieferten bis Ende 2016 genau 20 Teams, elf für die onkologische Indikation, neun für TBC. In 14 Teams aus der Umfragegruppe sind Vertragsärzte vertreten. Auffällig sei, dass in knapp der Hälfte aller ASV-Kernteams Ärzte aus einem Medizinischen Versorgungszentrum in vertragsärztlicher oder gemischter Trägerschaft waren.

\section{Keine Rede von flächendeckender Etablierung}

Lediglich zwei Teamleiter haben reine Klinikteams zusammengestellt. Für die onkologische Indikation ist die Zusammenarbeit über die Sektorengrenzen hinweg gesetzlich vorgeschrieben. Von einer flächendeckenden Etablierung der ASV könne nach wie vor keine Rede sein, schreiben die Studienautoren. Im für die Auswertung betrachteten zweiten Quartal 2016 behandelten die ASV-Teams für gastrointestinale Tumoren im Schnitt 61 Patienten, jene für die Tbc 26.

Das Anzeigeverfahren und die Abrechnung werden von den Beteiligten als bürokratisch erlebt. Kritisiert wird zum Beispiel der Umfang der einzureichenden Unterlagen. Dafür seien zum Teil auch die Teamgrößen von bis zu 50 teilnehmenden Ärzten verantwortlich, heißt es beim ASV-Bundesverband. Gerechnet hat sich die Teilnahme für die Ärzte nach deren Angaben bislang kaum. In der ASV sehen aber vor allem die Vertragsärzte langfristige strategische Vorteile. 